\title{
COMPARISON OF HAUSDORFF MEASURES WITH RESPECT TO THE EUCLIDEAN AND THE HEISENBERG METRIC
}

\author{
Zoltán M. Balogh, Matthieu Rickly AND \\ Francesco Serra Cassano*
}

\begin{abstract}
We compare the Hausdorff measures and dimensions with respect to the Euclidean and Heisenberg metrics on the first Heisenberg group. The result is a dimension jump described by two inequalities. The sharpness of our estimates is shown by examples. Moreover a comparison between Euclidean and $\mathbb{H}-$-rectifiability is given.
\end{abstract}

\section{Introduction}

In this paper we consider the Heisenberg group $\mathbb{H}=\mathbb{H}^{1}=\left(\mathbb{R}^{3}, *\right)$ as a homogeneous group endowed with the left invariant, homogeneous Heisenberg distance $d_{H}$ defined as follows. The group multiplication $*: \mathbb{H} \times \mathbb{H} \rightarrow \mathbb{H}$ is given by

$$
(x, y, t) *\left(x^{\prime}, y^{\prime}, t^{\prime}\right):=\left(x+x^{\prime}, y+y^{\prime}, t+t^{\prime}+2\left(x^{\prime} y-y^{\prime} x\right)\right) .
$$

$\mathbb{H}$ is endowed with the homogeneous norm

$$
\|p\|_{H}:=\left(\left(x^{2}+y^{2}\right)^{2}+t^{2}\right)^{\frac{1}{4}}
$$

if $p=(x, y, t) \in \mathbb{H}$, which induces the Heisenberg distance

$$
d_{H}\left(p, p^{\prime}\right):=\left\|p^{-1} * p^{\prime}\right\|_{H} .
$$

It is well-known that the topological dimension of $\mathbb{H}$ is 3 , since $\mathbb{H}$ coincides with $\mathbb{R}^{3}$ as a smooth manifold (see Lemma 2.1). On the other hand, the Hausdorff measures and dimensions of subsets of $\mathbb{H} \equiv \mathbb{R}^{3}$ with respect to either $d_{H}$ or the Euclidean metric $d_{E}$ can be very different. For

2000 Mathematics Subject Classification. 28A78, 43A80.

Key words. Hausdorff measures, Hausdorff dimension, Heisenberg metric.

${ }^{*}$ F.S.C. is supported by GNAMPA of INDAM, project "Analysis in metric spaces and subelliptic equations", by MURST, Italy, and University of Trento, Italy. Part of the work was done while F.S.C. was a visitor at the University of Bern. He wishes to thank the Institute of Mathematics for its hospitality. 
instance, the Hausdorff dimension of $\left(\mathbb{R}^{3}, d_{H}\right)$ is 4 , while the dimension of a regular surface in $\left(\mathbb{R}^{3}, d_{H}\right)$ is 3 . However, if we consider a regular curve in $\left(\mathbb{R}^{3}, d_{H}\right)$, then it may have Hausdorff dimension both 1 and 2 (see [Gro] and also $[\mathbf{S t r}]$ ).

The purpose of this paper is to describe in details this dimension jump phenomenon between the Heisenberg and the Euclidean Hausdorff dimensions on subsets of $\mathbb{R}^{3}$. Indeed we perform a comparison between the $\alpha$-dimensional Hausdorff measures induced on $\mathbb{R}^{3}$ by $d_{H}$ and $d_{E}$, which we respectively denote with $\mathcal{H}_{H}^{\alpha}$ and $\mathcal{H}_{E}^{\alpha}$. More precisely our first result reads as follows:

Theorem 1.1 (Dimension jump theorem). Let $\alpha \geq 0$. Then

$$
\mathcal{H}_{H}^{\min \{2 \alpha, \alpha+1\}} \ll \mathcal{H}_{E}^{\alpha},
$$

i.e. $\mathcal{H}_{H}^{\min \{2 \alpha, \alpha+1\}}$ is absolutely continuous with respect to $\mathcal{H}_{E}^{\alpha}$.

$$
\mathcal{H}_{E}^{\min \left\{\alpha, 1+\frac{\alpha}{2}\right\}} \ll \mathcal{H}_{H}^{\alpha},
$$

i.e. $\mathcal{H}_{E}^{\min \left\{\alpha, 1+\frac{\alpha}{2}\right\}}$ is absolutely continuous with respect to $\mathcal{H}_{H}^{\alpha}$.

Our second result shows that the estimates of Theorem 1.1 are sharp:

Theorem 1.2 (Sharpness of the dimension jump).

(i) Given $0<\alpha \leq 3$, there is a compact subset $A_{\alpha}$ of $\mathbb{H}$ satisfying

$$
\mathcal{H}_{E}^{\alpha}\left(A_{\alpha}\right)<\infty \quad \text { and } \mathcal{H}_{H}^{\min \{2 \alpha, \alpha+1\}}\left(A_{\alpha}\right)>0 .
$$

(ii) For $0<\alpha<2$ and $\alpha=4$, there is a compact subset $A_{\alpha}$ of $\mathbb{H}$ satisfying

$$
\mathcal{H}_{H}^{\alpha}\left(A_{\alpha}\right)<\infty \quad \text { and } \quad \mathcal{H}_{E}^{\min \left\{\alpha, 1+\frac{\alpha}{2}\right\}}\left(A_{\alpha}\right)>0 .
$$

For $2 \leq \alpha<4$ and $0<\delta<1$, there is a compact subset $A_{\alpha, \delta}$ of $\mathbb{H}$ satisfying

$$
\mathcal{H}_{H}^{\alpha}\left(A_{\alpha, \delta}\right)=0 \quad \text { and } \quad \mathcal{H}_{E}^{\min \left\{\alpha, 1+\frac{\alpha}{2}\right\}-\delta}\left(A_{\alpha, \delta}\right)=\mathcal{H}_{E}^{1+\frac{\alpha}{2}-\delta}\left(A_{\alpha, \delta}\right)>0 .
$$

Remark 1.1. We conjecture that given $2 \leq \alpha<4$, there is a compact subset $A_{\alpha}$ of $\mathbb{H}$ such that

$$
\mathcal{H}_{E}^{\min \left\{\alpha, 1+\frac{\alpha}{2}\right\}}\left(A_{\alpha}\right)=\mathcal{H}_{E}^{1+\frac{\alpha}{2}}\left(A_{\alpha}\right)>0 \quad \text { and } \quad \mathcal{H}_{H}^{\alpha}\left(A_{\alpha}\right)<\infty .
$$

However, we have not yet been able to construct such sets. 
The technique involved in the proof of Theorem 1.1 is based on an optimal covering of Heisenberg balls by smaller Euclidean balls and viceversa. This kind of mutual coverings have already been proposed in [Gro]. The proof of Theorem 1.2 relies on some more delicate arguments involving recent results on the size of Cantor-type and characteristic sets of regular surfaces in the metric spaces $\left(\mathbb{R}^{3}, d_{H}\right)$ and $\left(\mathbb{R}^{3}, d_{E}\right)$ (see $[\mathbf{B a 1}]$, $[\mathrm{Ba} 2])$.

Another striking feature of the Heisenberg group is the lack of $m$-rectifiable subsets in the metric space $\left(\mathbb{H}, d_{H}\right)$ according to the classical notion of rectifiability in a metric space due to Federer $[\mathbf{F e}]$. Indeed, it has been proved that $\left(\mathbb{H}, d_{H}\right)$ is purely $m$-unrectifiable for $m=2,3,4$ (see [AK, Theorem 7.2]). This has led to a more intrinsic definition of rectifiability in $\left(\mathbb{H}, d_{H}\right)$, the 3-dimensional $\mathbb{H}$-rectifiability, introduced in [FSSC1]. We will use our results from Theorem 1.2 to compare the classical notion of 2-Euclidean rectifiability in $\left(\mathbb{R}^{3}, d_{E}\right)$ to the notion of 3-dimensional $\mathbb{H}$-rectifiability (see Theorem 5.1).

The paper is organized as follows: in Section 2 we recall some basic facts about the Heisenberg group and the Hausdorff measures defined on it. In Section 3 we prove our first theorem. The second theorem is proved in Section 4. In the last section we apply our results to compare 2-rectifiable subsets of $\left(\mathbb{H}, d_{E}\right)$ and 3-dimensional $\mathbb{H}$-rectifiable subsets of $\left(\mathbb{H}, d_{H}\right)$.

Acknowledgements. We thank Pertti Mattila for useful discussions on the subject.

\section{Basic definitions and preliminary results}

In this section, we briefly restate the basic definitions and results needed in the rest of the article.

We use the model of the first Heisenberg group $\mathbb{H}=\mathbb{H}^{1}$ with underlying space $\mathbb{R}^{3}$ and group operation given by (1.1). Notice that $p^{-1}=-p$ and that $0=(0,0,0)$ is the unit element of the group $\mathbb{H}$. The dilation by $r$ is the automorphism $\delta_{r}: \mathbb{H} \rightarrow \mathbb{H}$ given by

$$
\delta_{r}(x, y, t):=\left(r x, r y, r^{2} t\right) .
$$

For $p \in \mathbb{H}$ the left translation by $p$ is the automorphism $l_{p}: \mathbb{H} \rightarrow \mathbb{H}$ defined by

$$
l_{p}\left(p^{\prime}\right):=p * p^{\prime} .
$$


The 3-dimensional Lebesgue measure $\mathcal{L}^{3}$ in $\mathbb{R}^{3} \equiv \mathbb{H}$ is a bi-invariant Haar measure for the group and satisfies $\mathcal{L}^{3}\left(\delta_{r}(A)\right)=r^{4} \mathcal{L}^{3}(A)$ whenever $A \subseteq \mathbb{H}$ and $r>0$ (see [FoSt, Proposition 1.2 (c)]).

The Lie algebra of $\mathbb{H}$ is spanned by the left invariant vector fields

$$
X=\frac{\partial}{\partial x}+2 y \frac{\partial}{\partial t}, \quad Y=\frac{\partial}{\partial y}-2 x \frac{\partial}{\partial t}, \quad T=\frac{\partial}{\partial t} .
$$

The Lie algebra structure of $\mathbb{H}$ is determined by the only non-trivial commutator relation $[X, Y]=-4 T$.

The vector fields $X$ and $Y$ span a vector bundle, the so-called horizontal bundle $\mathrm{HH}$, where $\mathrm{HH}_{p}:=\operatorname{span}\{X(p), Y(p)\}$ for all $p \in \mathbb{H}$, which can be canonically identified with a vector subbundle of the tangent vector bundle $\mathrm{TH} \equiv \mathrm{TR}^{3}$.

The following relationship between the distances $d_{E}$ and $d_{H}$ in $\mathbb{R}^{3}$ can be easily verified.

Lemma 2.1. Let $A$ be a bounded subset of $\left(\mathbb{R}^{3}, d_{E}\right)$ and let $b \geq 1$ be $a$ bound for $A$ with respect to $d_{E}$. Then there is a positive constant $c=c(b)$ such that for $p, p^{\prime} \in A$ we have

$$
\frac{1}{c} d_{E}\left(p, p^{\prime}\right) \leq d_{H}\left(p, p^{\prime}\right) \leq c\left(d_{E}\left(p, p^{\prime}\right)\right)^{\frac{1}{2}} .
$$

In particular the identity map $\mathrm{Id}:\left(\mathbb{R}^{3}, d_{E}\right) \rightarrow\left(\mathbb{R}^{3}, d_{H}\right)$ is a homeomorphism.

In the sequel, $B_{E}(p, r)$ and $B_{H}(p, r)$ will denote the closed ball of radius $r>0$ centered at $p$ in $\left(\mathbb{H}, d_{E}\right)$ and $\left(\mathbb{H}, d_{H}\right)$ respectively. $\|\cdot\|$ will denote the Euclidean norm on $\mathbb{R}^{n}$ for $n=2,3$.

Let us now recall the definition of Hausdorff and spherical measures on a metric space $(X, d)$. Given a subset $A$ of $X$, the diameter of $A$ is

$$
\operatorname{diam}_{d}(A):=\sup \left\{d\left(a, a^{\prime}\right) \mid a, a^{\prime} \in A\right\} .
$$

We write diam instead of $\operatorname{diam}_{d}$ when there is no risk of confusion, and we let $\operatorname{diam}_{E}:=\operatorname{diam}_{d_{E}}$ and $\operatorname{diam}_{H}:=\operatorname{diam}_{d_{H}}$. Observe that for $p \in \mathbb{H}$ and $r>0$ we have

$$
\operatorname{diam}_{H}\left(B_{H}(x, r)\right)=2 r .
$$


For $0 \leq s<\infty, 0<\delta \leq \infty$ and $A \subseteq X$, we let

$\mathcal{H}_{d, \delta}^{s}(A):=\inf \left\{\sum_{n=1}^{\infty}\left(\operatorname{diam}\left(E_{n}\right)\right)^{s} \mid E_{n} \subseteq X, \operatorname{diam}\left(E_{n}\right) \leq \delta, A \subseteq \bigcup_{n \in \mathbb{N}} E_{n}\right\}$

and

$$
\begin{array}{r}
\mathcal{S}_{d, \delta}^{s}(A):=\inf \left\{\sum_{n=1}^{\infty} \operatorname{diam}\left(B\left(x_{n}, r_{n}\right)\right)^{s} \mid \operatorname{diam}\left(B\left(x_{n}, r_{n}\right)\right) \leq \delta,\right. \\
\left.A \subseteq \bigcup_{n \in \mathbb{N}} B\left(x_{n}, r_{n}\right)\right\},
\end{array}
$$

where $B(x, r)$ denotes the closed ball of radius $r$ centered at $x$. Since $0<$ $\delta_{1} \leq \delta_{2}$ clearly implies $\mathcal{H}_{d, \delta_{2}}^{s}(A) \leq \mathcal{H}_{d, \delta_{1}}^{s}(A)$ and $\mathcal{S}_{d, \delta_{2}}^{s}(A) \leq \mathcal{S}_{d, \delta_{1}}^{s}(A)$, we may define the $s$-dimensional Hausdorff measure $\mathcal{H}_{d}^{s}$ on $(X, d)$ and the $s$-dimensional spherical measure $\mathcal{S}_{d}^{s}$ on $(X, d)$ by

$$
\mathcal{H}_{d}^{s}(A):=\lim _{\delta \downarrow 0} \mathcal{H}_{d, \delta}^{s}(A)
$$

and

$$
\mathcal{S}_{d}^{s}(A):=\lim _{\delta \downarrow 0} \mathcal{S}_{d, \delta}^{s}(A)
$$

respectively.

We will write $\mathcal{H}_{E}^{s}, \mathcal{S}_{E}^{s}$ instead of $\mathcal{H}_{d_{E}}^{s}, \mathcal{S}_{d_{E}}^{s}$ and $\mathcal{H}_{H}^{s}, \mathcal{S}_{H}^{s}$ instead of $\mathcal{H}_{d_{H}}^{s}, \mathcal{S}_{d_{H}}^{s}$ and we will generally consider these measures on $\mathbb{R}^{3}$. By abuse of notation we shall also write $\mathcal{H}_{E}^{s}$ for the $s$-dimensional Hausdorff measure on $\left(\mathbb{R}^{m}, d_{E}\right)$ for $m=1,2$.

The Hausdorff dimension of a set $A \subseteq(X, d)$ is

$$
\mathcal{H}-\operatorname{dim}_{d}(A):=\inf \left\{s \geq 0 \mid \mathcal{H}_{d}^{s}(A)=0\right\} .
$$

In the following proposition we collect some general properties of Hausdorff measures that will be needed below.

\section{Proposition 2.2.}

(i) Let $(X, d)$ be a metric space. Then $\mathcal{H}_{d}^{s}$ and $\mathcal{S}_{d}^{s}$ are regular Borel (outer) measures and

$$
\mathcal{H}_{d}^{s}(A) \leq \mathcal{S}_{d}^{s}(A) \leq 2^{s} \mathcal{H}_{d}^{s}(A)
$$

for all $A \subseteq X, s \geq 0$. 
(ii) Let $\left(X_{i}, d_{i}\right)(i=1,2)$ be two metric spaces and let $f:\left(X_{1}, d_{1}\right) \rightarrow$ $\left(X_{2}, d_{2}\right)$ be a L-Lipschitz continuous map, i.e.

$$
d_{2}(f(x), f(y)) \leq L \cdot d_{1}(x, y) \quad\left(\forall x, y \in X_{1}\right) .
$$

Then

$$
\mathcal{H}_{d_{2}}^{s}(f(A)) \leq L^{s} \cdot \mathcal{H}_{d_{1}}^{s}(A)
$$

for all $A \subseteq X_{1}, s \geq 0$.

(iii) Let $(X, d)$ be a metric space and let $d_{t}(x, y):=(d(x, y))^{t}$ for $x, y \in$ $X$ and $t \in(0,1)$. Then $d_{t}$ is a distance on $X$ and

$$
\mathcal{H}_{d}^{s}(A)=\mathcal{H}_{d_{t}}^{\frac{s}{t}}(A)
$$

for all $A \subseteq X, s \geq 0$ and $t \in(0,1)$.

(iv) Let $(X, d)$ be a metric space and let $Y \subseteq X$. Denote by $d_{Y}$ the metric on $Y$ induced by $d$. Then

$$
\mathcal{H}_{d}^{s}(A)=\mathcal{H}_{d_{Y}}^{s}(A)
$$

for all $A \subseteq Y, s \geq 0$.

(v) Properties (ii) and (iii) hold if we replace the Hausdorff measure $\mathcal{H}_{d}^{s}$ by the spherical measure $\mathcal{S}_{d}^{s}$.

Proof: All these statements are classical and can be found in $[\mathbf{F a}],[\mathbf{F e}]$ or $[\mathbf{M a}]$.

Remark 2.1. Using (ii) of the above proposition, one easily sees that if $d_{1}$ and $d_{2}$ are two bi-Lipschitz equivalent metrics on $X$, then $\mathcal{H}_{d_{1}}^{s}$ and $\mathcal{H}_{d_{2}}^{s}$ are mutually absolutely continuous measures. In particular, if $A \subseteq X$ then $\mathcal{H}-\operatorname{dim}_{d_{1}}(A)=\mathcal{H}-\operatorname{dim}_{d_{2}}(A)$.

Using the homogeneity and left invariance of $d_{H}$, one has

$$
\mathcal{H}_{H}^{s}\left(l_{p}(A)\right)=\mathcal{H}_{H}^{s}(A) \text { and } \mathcal{H}_{H}^{s}\left(\delta_{r}(A)\right)=r^{s} \mathcal{H}_{H}^{s}(A)
$$

for any $A \subseteq \mathbb{H}, p \in \mathbb{H}, s \geq 0$ and $r>0$. Moreover it is easy to see that $\mathcal{H}_{H}^{4}=c \mathcal{L}^{3}$ for a suitable positive constant $c$.

In Subsection 4.1 we will make use of the following criterion giving a lower bound on the Hausdorff dimension of a set in a metric space.

Lemma 2.3. Let $(X, d)$ be a separable metric space, let $A \subseteq X$ and let $s>0$. Suppose there is a measure $\mu$ on $X$ with the following properties:

(i) $\mu(A)>0$.

(ii) $\mu(B(x, r) \cap A) \leq c_{\mu} r^{s}$ for all $x \in X$ and $0<r \leq R$, where $c_{\mu}$, $R>0$ are fixed constants.

Then $\mathcal{H}_{d}^{s}(A)>0$.

The proof is a simple exercise. 


\section{Proof of the dimension jump theorem}

In the following we prove the absolute continuity of $\mathcal{H}_{H}^{2 \alpha}$ and $\mathcal{H}_{H}^{\alpha+1}$ with respect to $\mathcal{H}_{E}^{\alpha}$ (Propositions 3.2 (i) and 3.6 (i) below) and the absolute continuity of $\mathcal{H}_{E}^{\alpha}$ and $\mathcal{H}_{E}^{1+\frac{\alpha}{2}}$ with respect to $\mathcal{H}_{H}^{\alpha}$ (Propositions 3.2 (ii) and 3.6 (ii) below). Indeed, notice that from Proposition 2.2 (i) we can indifferently estimate the Hausdorff or spherical measures. We start with a technical lemma:

Lemma 3.1. A subset $A$ of $\mathbb{H}$ is bounded with respect to $d_{E}$ if and only if it is bounded with respect to $d_{H}$. More precisely, if $b_{E} \geq 1$ is a bound for $A$ with respect to $d_{E}$, then $2^{\frac{1}{4}} b_{E}$ is a bound for $A$ with respect to $d_{H}$, and if $b_{H} \geq 1$ is a bound for $A$ with respect to $d_{H}$, then $2^{\frac{1}{2}} b_{H}^{2}$ is a bound for $A$ with respect to $d_{E}$.

The proof is left to the reader.

Proposition 3.2. The Hausdorff measures satisfy the following absolute continuity properties ( $\alpha \geq 0$ arbitrary):

(i) $\mathcal{H}_{H}^{\alpha} \ll \mathcal{H}_{E}^{\frac{\alpha}{2}}$.

(ii) $\mathcal{H}_{E}^{\alpha} \ll \mathcal{H}_{H}^{\alpha}$.

Proof: (i) Suppose that $\mathcal{H}_{E}^{\frac{\alpha}{2}}(A)=0$ and denote by $\sqrt{d_{E}}: \mathbb{R}^{3} \times \mathbb{R}^{3} \rightarrow$ $[0,+\infty)$ the distance $\sqrt{d_{E}}\left(p, p^{\prime}\right):=\left(d_{E}\left(p, p^{\prime}\right)\right)^{\frac{1}{2}}$ if $p, p^{\prime} \in \mathbb{R}^{3}$. Then by Proposition 2.2 (iii) with $t=\frac{1}{2}$ we also get $0=\mathcal{H}_{E}^{\frac{\alpha}{2}}(A)=\mathcal{H}_{\sqrt{d_{E}}}^{\alpha}(A)$. Let $A_{n}:=A \cap B_{E}(0, n)$. Applying Lemma 2.1 with $A=A_{n}$, there is a positive constant $c_{n}$ depending only on $n$, such that for all $p, p^{\prime} \in A_{n}$ we have

$$
\frac{1}{c_{n}} d_{E}\left(p, p^{\prime}\right) \leq d_{H}\left(p, p^{\prime}\right) \leq c_{n} \sqrt{d_{E}}\left(p, p^{\prime}\right) .
$$

Let $d_{H}^{(n)}$ and $\sqrt{d_{E}^{(n)}}$ denote the restrictions of the distances $d_{H}$ and $\sqrt{d_{E}}$ to $A_{n}$. By the right inequality in (3.1) and Proposition 2.2 (ii) with $X_{1}=X_{2}=A_{n}, f=\mathrm{Id}, d_{1}=\sqrt{d_{E}^{(n)}}, d_{2}=d_{H}^{(n)}$ and $L=c_{n}$

$$
\mathcal{H}_{d_{H}^{(n)}}^{\alpha}\left(A_{n}\right) \leq c_{n}^{\alpha} \mathcal{H}_{\sqrt{d_{E}^{(n)}}}^{\alpha}\left(A_{n}\right) .
$$

On the other hand from Proposition 2.2 (iv)

$$
\mathcal{H}_{H}^{\alpha}\left(A_{n}\right)=\mathcal{H}_{d_{H}^{(n)}}^{\alpha}\left(A_{n}\right) \leq c_{n}^{\alpha} \mathcal{H}_{\sqrt{d_{E}^{(n)}}}^{\alpha}\left(A_{n}\right)=\mathcal{H}_{\sqrt{d_{E}}}^{\alpha}\left(A_{n}\right)=0 .
$$

Then, by passing to the limit when $n \rightarrow \infty$, we get $\mathcal{H}_{H}^{\alpha}(A)=0$. 
The statement (ii) can be proved by similar arguments using the left inequality in (3.1).

In the following we shall prove $\mathcal{S}_{H}^{\alpha+1} \ll \mathcal{S}_{E}^{\alpha}$ and $\mathcal{S}_{E}^{1+\frac{\alpha}{2}} \ll \mathcal{S}_{H}^{\alpha}$, cf. Proposition 3.6 below. This is considerably more complicated than Proposition 3.2. The proof is based on more involved covering lemmas relating the Euclidean and Heisenberg balls. The statements of these covering lemmas can be found in [Gro, 0.6.C].

Before going into details we need one more technical lemma in order to control the distortion of the shape of Euclidean balls under group translation.

Lemma 3.3. Let $A$ be a bounded subset of $\left(\mathbb{R}^{3}, d_{E}\right)$ with bound $b \geq 0$. For $r>0, p=(x, y, t) \in \mathbb{R}^{3}$ and $p^{\prime}=\left(x^{\prime}, y^{\prime}, t^{\prime}\right) \in A$ we have

$$
l_{p^{\prime}}\left(B_{E}(p, r)\right) \subseteq B_{E}\left(l_{p^{\prime}}(p),(2 b+1) r\right) .
$$

Moreover if $l_{p^{\prime}}(p)+\left(x^{\prime \prime}, y^{\prime \prime}, t^{\prime \prime}\right) \in l_{p^{\prime}}\left(B_{E}(p, r)\right)$ then $\left\|\left(x^{\prime \prime}, y^{\prime \prime}\right)\right\| \leq r$.

Proof: Let $\left(x+x^{\prime \prime \prime}, y+y^{\prime \prime \prime}, t+t^{\prime \prime \prime}\right) \in B_{E}(p, r)$.

$$
\begin{aligned}
& l_{p^{\prime}}\left(x+x^{\prime \prime \prime}, y+y^{\prime \prime \prime}, t+t^{\prime \prime \prime}\right) \\
& \quad=\left(x^{\prime}+x+x^{\prime \prime \prime}, y^{\prime}+y+y^{\prime \prime \prime}, t^{\prime}+t+t^{\prime \prime \prime}+2\left(\left(x+x^{\prime \prime \prime}\right) y^{\prime}-x^{\prime}\left(y+y^{\prime \prime \prime}\right)\right)\right) \\
& \quad=l_{p^{\prime}}(p)+\left(x^{\prime \prime \prime}, y^{\prime \prime \prime}, t^{\prime \prime \prime}+2\left(x^{\prime \prime \prime} y^{\prime}-x^{\prime} y^{\prime \prime \prime}\right)\right) .
\end{aligned}
$$

Now $\left\|\left(x^{\prime \prime}, y^{\prime \prime}\right)\right\|=\left\|\left(x^{\prime \prime \prime}, y^{\prime \prime \prime}\right)\right\| \leq r$ and

$$
\begin{aligned}
& \left\|\left(x^{\prime \prime \prime}, y^{\prime \prime \prime}, t^{\prime \prime \prime}+2\left(x^{\prime \prime \prime} y^{\prime}-x^{\prime} y^{\prime \prime \prime}\right)\right)\right\|^{2} \\
& \quad=x^{\prime \prime \prime}{ }^{2}+y^{\prime \prime \prime}{ }^{2}+\left|t^{\prime \prime \prime}+2\left(x^{\prime \prime \prime} y^{\prime}-x^{\prime} y^{\prime \prime \prime}\right)\right|^{2} \\
& \leq x^{\prime \prime \prime}{ }^{2}+y^{\prime \prime \prime}{ }^{2}+\left(\left|t^{\prime \prime \prime}\right|+2\left\|p^{\prime}\right\| \cdot\left\|\left(x^{\prime \prime \prime}, y^{\prime \prime \prime}, t^{\prime \prime \prime}\right)\right\|\right)^{2} \\
& \leq\left\|\left(x^{\prime \prime \prime}, y^{\prime \prime \prime}, t^{\prime \prime \prime}\right)\right\|^{2}+4 b\left\|\left(x^{\prime \prime \prime}, y^{\prime \prime \prime}, t^{\prime \prime \prime}\right)\right\| \cdot\left|t^{\prime \prime \prime}\right|+4 b^{2}\left\|\left(x^{\prime \prime \prime}, y^{\prime \prime \prime}, t^{\prime \prime \prime}\right)\right\|^{2} \\
& \leq r^{2}+4 b r^{2}+4 b^{2} r^{2}=(2 b+1)^{2} r^{2} .
\end{aligned}
$$

The claim follows.

The following statement exhibits a close-to-optimal covering of a Euclidean ball by Heisenberg balls. 
Proposition 3.4. Let $A$ be a bounded subset of $\left(\mathbb{R}^{3}, d_{E}\right)$ with bound $b \geq$ 0 . Then there exists $N=N(b) \in \mathbb{N}$ such that for any Euclidean ball $B_{E}(p, r)$ with $p \in A$ and $0<r<1$ we can find Heisenberg balls $B_{H}\left(p_{1}, r\right), \ldots, B_{H}\left(p_{k}, r\right)$ satisfying:

(i) $B_{E}(p, r) \subseteq \bigcup_{i=1}^{k} B_{H}\left(p_{i}, r\right)$.

(ii) $k \leq \frac{N}{r}$.

Remark 3.1. Notice that (ii) yields a sharp control on the number $k$ of Heisenberg balls needed to cover the Euclidean ball in terms of its radius $r$.

Proof: It is clearly enough to show that, given a bounded subset $A$ of $\left(\mathbb{R}^{3}, d_{E}\right)$ with bound $b \geq 0$, there is $N=N(b) \in \mathbb{N}$ such that for $p \in A$ and $0<r<1$ we can find $B_{H}\left(p_{1}, r\right), \ldots, B_{H}\left(p_{k}, r\right)$ satisfying:

$$
B_{E}(p, r / 2) \subseteq \bigcup_{i=1}^{k} B_{H}\left(p_{i}, r\right) \quad \text { and } \quad k \leq \frac{N}{r}
$$

The idea is to use a group translation to move the Euclidean ball to the origin and to perform the covering there with balls centered on the vertical axis $O_{t}$. For $p=(0,0, t) \in O_{t}$ and $r>0$, let us denote by $B_{H, \infty}(p, r)$ the set of points $p^{\prime}=\left(x^{\prime}, y^{\prime}, t^{\prime}\right) \in \mathbb{H}$ satisfying $\left\|\left(x^{\prime}, y^{\prime}\right)\right\| \leq r$ and $\left|t^{\prime}-t\right| \leq r^{2}$. Hence $B_{H, \infty}(p, r)$ is a flat box of height $2 r^{2}$ centered at $p \in \mathbb{H}$ and its orthogonal projection along the vertical axis is a disk of radius $r$. Now let $p \in A, 0<r<1$ and let $k:=\left[\frac{4 b+2}{r}\right]+1$. By Lemma 3.3 we can cover $l_{p^{-1}}\left(B_{E}\left(p, \frac{r}{2}\right)\right)$ with $k$ boxes $B_{H, \infty}\left(p_{i}, \frac{r}{2}\right)$, where $i \in\{1, \ldots, k\}$ and $\left(p_{i}\right)_{i \in\{1, \ldots, k\}}$ are suitable points on the vertical axis. The reader can easily see that $B_{H, \infty}\left(p_{i}, \frac{r}{2}\right) \subseteq B_{H}\left(p_{i}, r\right)$ for $1 \leq i \leq k$, thus

$$
B_{E}(p, r / 2) \subseteq l_{p}\left(\bigcup_{i=1}^{k} B_{H}\left(p_{i}, r\right)\right)=\bigcup_{i=1}^{k} l_{p}\left(B_{H}\left(p_{i}, r\right)\right)=\bigcup_{i=1}^{k}\left(B_{H}\left(p * p_{i}, r\right)\right) .
$$

Finally, $k r<\frac{4 b+3}{r} r=4 b+3$ and the claim is proved with $N:=[4 b+$ $3]+1$.

The following statement is in some sense the counterpart of Proposition 3.4. It presents a close-to-optimal covering of a Heisenberg ball by Euclidean balls. 
Proposition 3.5. Given a bounded subset $A$ of $\left(\mathbb{R}^{3}, d_{E}\right)$ with bound $b \geq$ 0 , there is $N=N(b) \in \mathbb{N}$ such that for any Heisenberg ball $B_{H}(p, r)$ with $p \in A$ and $0<r<1$ we can find $B_{E}\left(p_{1}, r^{2}\right), \ldots, B_{E}\left(p_{k}, r^{2}\right)$ satisfying:

(i) $B_{H}(p, r) \subseteq \bigcup_{i=1}^{k} B_{E}\left(p_{i}, r^{2}\right)$.

(ii) $k \leq \frac{N}{r^{2}}$.

Remark 3.2. Once again (ii) gives a sharp bound on the number $k$ of Euclidean balls needed to cover the Heisenberg ball in terms of its radius $r$.

Proof: First we consider Heisenberg balls centered at the origin. We will prove the following: There is $K \in \mathbb{N}$ such that given $0<r<1$ we can find $B_{E}\left(p_{1}, r^{2}\right), \ldots, B_{E}\left(p_{l}, r^{2}\right)$ satisfying:

$$
B_{H}(0, r) \subseteq \bigcup_{i=1}^{l} B_{E}\left(p_{i}, 3^{\frac{1}{2}} r^{2}\right) \quad \text { and } \quad l \leq \frac{K}{r^{2}} .
$$

Indeed, it is easy to verify that $B_{H}(0, r) \subseteq B_{H, \infty}(0, r)$ (where $B_{H, \infty}(0, r)$ is the box introduced in the proof of Proposition 3.4), and $B_{H, \infty}(0, r)$ is contained in a number $\left(\left[\frac{1}{r}\right]+1\right)^{2}$ of cubes of side length $2 r^{2}$, each of which is contained in a Euclidean ball of radius $3^{\frac{1}{2}} r^{2}$. Hence, the claim is proved with $K:=4$, since $\left(\left[\frac{1}{r}\right]+1\right)<\frac{2}{r}$. Now given $p \in A$ and $0<r<1$, we can find $B_{E}\left(p_{1}, 3^{\frac{1}{2}} r^{2}\right), \ldots, B_{E}\left(p_{l}, 3^{\frac{1}{2}} r^{2}\right)$ covering $l_{p^{-1}}\left(B_{H}(p, r)\right)=$ $B_{H}(0, r)$ so that $l r^{2} \leq K$. Clearly there is a constant $C=C(b)$ and balls $B_{E}\left(p_{1}, \frac{r^{2}}{2 b+1}\right), \ldots, B_{E}\left(p_{k}, \frac{r^{2}}{2 b+1}\right)$ covering $B_{H}(0, r)$ so that $k r^{2} \leq K \cdot C$. We let $N:=K \cdot C$, and with the help of Lemma 3.3 we compute:

$$
\begin{aligned}
B_{H}(p, r) & =l_{p}\left(l_{p^{-1}}\left(B_{H}(p, r)\right)\right) \subseteq l_{p}\left(\bigcup_{i=1}^{k} B_{E}\left(p_{i}, r^{2} /(2 b+1)\right)\right) \\
& =\bigcup_{i=1}^{k} l_{p}\left(B_{E}\left(p_{i}, r^{2} /(2 b+1)\right)\right) \subseteq \bigcup_{i=1}^{k} B_{E}\left(l_{p}\left(p_{i}\right), r^{2}\right) .
\end{aligned}
$$

Proposition 3.6. The spherical measures satisfy the following absolute continuity properties ( $\alpha \geq 0$ arbitrary):

(i) $\mathcal{S}_{H}^{\alpha+1} \ll \mathcal{S}_{E}^{\alpha}$.

(ii) $\mathcal{S}_{E}^{1+\frac{\alpha}{2}} \ll \mathcal{S}_{H}^{\alpha}$.

Proof: The proof is based on the covering statements formulated in Propositions 3.4 and 3.5. Let $\alpha \geq 0$ and $A \subseteq \mathbb{H}$. 
(i) Suppose that $\mathcal{S}_{E}^{\alpha}(A)=0$. Let $A_{n}:=A \cap B_{E}(0, n)$ for all $n \in \mathbb{N}$. Given $n \in \mathbb{N}, 0<\delta<1$ and $\epsilon>0$ arbitrary, there is a covering of $A_{n}$ with Euclidean balls $\left(B_{E}\left(p_{i}, r_{i}\right)\right)_{i \in \mathbb{N}}$ such that $r_{i} \leq \frac{\delta}{2}, p_{i} \in B_{E}(0, n+1)$ for $i \in \mathbb{N}$ and

$$
\sum_{i=1}^{\infty}\left(2 r_{i}\right)^{\alpha} \leq \frac{\epsilon}{2 N(n+1)}
$$

where $N(n+1)$ is from Proposition 3.4. By Proposition 3.4, each $B_{E}\left(p_{i}, r_{i}\right)$ can be covered with Heisenberg balls $B_{H}\left(p_{i, j}, r_{i}\right)$, where $j \in\{1, \ldots, k(i)\}$ and $k(i) r_{i} \leq N(n+1)$. The resulting collection $\left(B_{H}\left(p_{i, j}, r_{i}\right)\right)_{i \in \mathbb{N}, j \in\{1, \ldots, k(i)\}}$ forms a covering of $A_{n}$. Now, for $m \in \mathbb{N}$,

$$
\sum_{i=1}^{m} \sum_{j=1}^{k(i)}\left(2 r_{i}\right)^{\alpha+1}=\sum_{i=1}^{m}\left(2 r_{i}\right)^{\alpha} 2 \sum_{j=1}^{k(i)} r_{i} \leq 2 N(n+1) \sum_{i=1}^{m}\left(2 r_{i}\right)^{\alpha} \leq \epsilon .
$$

Consequently, by $(2.3)$ we obtain $\mathcal{S}_{H, \delta}^{\alpha+1}\left(A_{n}\right) \leq \epsilon$. Since $0<\delta<1$ and $\epsilon>0$ are arbitrary, we get $\mathcal{S}_{H}^{\alpha+1}\left(A_{n}\right)=0$. Finally,

$$
\mathcal{S}_{H}^{\alpha+1}(A)=\mathcal{S}_{H}^{\alpha+1}\left(\bigcup_{n \in \mathbb{N}} A_{n}\right) \leq \lim _{n \rightarrow \infty} \mathcal{S}_{H}^{\alpha+1}\left(A_{n}\right)=0 .
$$

(ii) Suppose that $\mathcal{S}_{H}^{\alpha}(A)=0$. Let $A_{n}:=A \cap B_{H}(0, n)$ for all $n \in \mathbb{N}$. Given $n \in \mathbb{N}, 0<\delta<1$ and $\epsilon>0$ arbitrary, there is a covering of $A_{n}$ with Heisenberg balls $\left(B_{H}\left(p_{i}, r_{i}\right)\right)_{i \in \mathbb{N}}$ such that $r_{i} \leq \frac{\delta}{2}, p_{i} \in B_{H}(0, n+1) \subseteq$ $B_{E}\left(0,2^{\frac{1}{2}}(n+1)^{2}\right)$ for $i \in \mathbb{N}$ (see Lemma 3.1) and

$$
\sum_{i=1}^{\infty}\left(2 r_{i}\right)^{\alpha} \leq \frac{\epsilon}{2^{1-\frac{\alpha}{2}} N\left(2^{\frac{1}{2}}(n+1)^{2}\right)},
$$

where $N\left(2^{\frac{1}{2}}(n+1)^{2}\right)$ is from Proposition 3.5. By Proposition 3.5, each $B_{H}\left(p_{i}, r_{i}\right)$ can be covered with balls $B_{E}\left(p_{i, j}, r_{i}^{2}\right)$, where $j \in\{1, \ldots, k(i)\}$ and $k(i) r_{i}^{2} \leq N\left(2^{\frac{1}{2}}(n+1)^{2}\right)$. The resulting collection $\left(B_{E}\left(p_{i, j}, r_{i}^{2}\right)\right)_{i \in \mathbb{N}, j \in\{1, \ldots, k(i)\}}$ forms a covering of $A_{n}$. Now, for $m \in \mathbb{N}$,

$$
\sum_{i=1}^{m} \sum_{j=1}^{k(i)}\left(2 r_{i}^{2}\right)^{1+\frac{\alpha}{2}}=\sum_{i=1}^{m} 2^{1+\frac{\alpha}{2}} r_{i}^{\alpha} \sum_{j=1}^{k(i)} r_{i}^{2}=2^{1-\frac{\alpha}{2}} \sum_{i=1}^{m}\left(2 r_{i}\right)^{\alpha} \sum_{j=1}^{k(i)} r_{i}^{2}
$$

and

$$
2^{1-\frac{\alpha}{2}} \sum_{i=1}^{m}\left(2 r_{i}\right)^{\alpha} \sum_{j=1}^{k(i)} r_{i}^{2} \leq 2^{1-\frac{\alpha}{2}} N\left(2^{\frac{1}{2}}(n+1)^{2}\right) \sum_{i=1}^{m}\left(2 r_{i}\right)^{\alpha} \leq \epsilon .
$$


Consequently, $\mathcal{S}_{E, \delta}^{1+\frac{\alpha}{2}}\left(A_{n}\right) \leq \epsilon$. Since $0<\delta<1$ and $\epsilon>0$ are arbitrary, we get $\mathcal{S}_{E}^{1+\frac{\alpha}{2}}\left(A_{n}\right)=0$. Finally,

$$
\mathcal{S}_{E}^{1+\frac{\alpha}{2}}(A)=\mathcal{S}_{E}^{1+\frac{\alpha}{2}}\left(\bigcup_{n \in \mathbb{N}} A_{n}\right) \leq \lim _{n \rightarrow \infty} \mathcal{S}_{E}^{1+\frac{\alpha}{2}}\left(A_{n}\right)=0 .
$$

It is clear that Propositions 3.2 and 3.6 together with Proposition 2.2 (i) imply Theorem 1.1.

\section{Proof of the sharpness of the dimension jump}

Let us start by presenting the intuitive background behind the proof of Theorem 1.2. Observe first that a Heisenberg ball $B_{H}(p, r)$ for small $r>0$ has the following crucial property: in the directions of the horizontal space $\mathrm{H}_{p} \mathbb{H}$, the size of $B_{H}(p, r)$ is the same as for a Euclidean ball. In the complementary direction however, the size of $B_{H}(p, r)$ is much smaller: it is comparable to $r^{2}$. For the first statement of Theorem 1.2 we have to construct a set whose Heisenberg Hausdorff dimension and measure is as large as possible in comparison to its Euclidean Hausdorff dimension and measure. For this reason, the sets we construct will be in some sense distributed along the direction in which the Heisenberg balls are small: i.e. transversal to the horizontal plane distribution. For the second statement of Theorem 1.2 we have to do the opposite: we have to construct sets with possibly small Heisenberg-Hausdorff dimension and measure as related to the Euclidean Hausdorff dimension and measure. This sets will be in some sense tangent to the horizontal plane distribution.

Some of the sets constructed are of product-type. Recent results about the Hausdorff measure and dimension of such sets are due to Howroyd (see $[\mathbf{H o w}]$ ). However, the Heisenberg metric is not of product-type and therefore we cannot simply take over the results in [How], but we have to use many ad hoc arguments in our proofs instead.

One of the main building blocks needed in the construction of the sets mentioned in Theorem 1.2 is the Cantor-set $C_{s} \subseteq[0,1]$ of Hausdorff dimension $s$, where $0<s<1$. The construction is standard, nevertheless we shall recall it as we need its details in our proofs. Let us choose $\lambda>0$ such that $\lambda^{s}=\frac{1}{2}$. $C_{s}$ is obtained as the intersection $C_{s}=\bigcap_{k \in \mathbb{N}_{0}} C_{s}^{k}$, where for each $k \in \mathbb{N}_{0} C_{s}^{k}=\bigcup_{l=1}^{2^{k}} I_{k, l}$ is the union of $2^{k}$ pairwise disjoint intervals $I_{k, l}$ of diameter $\operatorname{diam}\left(I_{k, l}\right)=\lambda^{k}$ constructed recursively in the following way: we let $I_{0,1}=[0,1]$, and, given $k \in \mathbb{N}_{0}, 1 \leq l \leq 2^{k}$ 
and $I_{k, l}=\left[a_{k, l}, b_{k, l}\right]$, we let $I_{k+1,2 l-1}=\left[a_{k, l}, a_{k, l}+\lambda^{k+1}\right]$ and $I_{k+1,2 l}=$ $\left[b_{k, l}-\lambda^{k+1}, b_{k, l}\right]$.

We collect some standard facts (see [Fa] and [Ma, 4.10]) about $C_{s}$ in the following proposition:

\section{Proposition 4.1.}

(i) $C_{s}$ is a compact subset of $\mathbb{R}$ without interior points.

(ii) $0<\mathcal{H}_{E}^{s}\left(C_{s}\right)<\infty$.

\subsection{Sharpness of $\mathcal{H}_{H}^{\min \{2 \alpha, \alpha+1\}} \ll \mathcal{H}_{E}^{\alpha}$.}

We distinguish several cases depending on the magnitude of $\alpha>0$.

1. Case $0<\boldsymbol{\alpha} \leq$ 1. We let $A_{\alpha}:=\left\{(0,0, t) \mid t \in C_{\alpha}\right\}$ for $0<$ $\alpha<1$, where $C_{\alpha}$ is the Cantor set of dimension $\alpha$ constructed above, and $A_{1}:=\{(0,0, t) \mid t \in[0,1]\}$. By Proposition 4.1, we know that $0<\mathcal{H}_{E}^{\alpha}\left(A_{\alpha}\right)<\infty$. Let us observe that the restriction of $d_{H}$ to the vertical axis $O_{t}$ satisfies $d_{H}\left(p_{1}, p_{2}\right)=\left(d_{E}\left(p_{1}, p_{2}\right)\right)^{\frac{1}{2}}$ for $p_{1}, p_{2} \in O_{t}$. Since $A_{\alpha} \subseteq O_{t}$ and $\mathcal{H}_{E}^{\alpha}\left(A_{\alpha}\right)>0, \mathcal{H}_{H}^{2 \alpha}\left(A_{\alpha}\right)>0$ follows immediately from Proposition 2.2 (iii) and (iv).

2. Case $1<\boldsymbol{\alpha}<2 . A_{\alpha}$ is now a Cantor set of vertical segments given by $A_{\alpha}=\left\{(x, 0, t) \mid x \in C_{\alpha-1}, t \in[0,1]\right\}$.

First, we prove that $\mathcal{H}_{E}^{\alpha}\left(A_{\alpha}\right)<\infty$. Since we are working with the Cantor set $C_{\alpha-1}$, we have $\lambda^{\alpha-1}=\frac{1}{2}$. Let $\delta>0$ arbitrary and let $k \in \mathbb{N}$ such that $2^{\frac{1}{2}} \lambda^{k}<\delta$. Consider the sets $J_{l, m}:=I_{k, l} \times\{0\} \times$ $\left[m \lambda^{k},(m+1) \lambda^{k}\right]$, where $l=1, \ldots, 2^{k}$ and $m=0, \ldots,\left[\lambda^{-k}+1\right]-1$. Then $A_{\alpha} \subseteq \bigcup_{l, m} J_{l, m}$, and

$\mathcal{H}_{E, \delta}^{\alpha}\left(A_{\alpha}\right) \leq \sum_{l, m}\left(\operatorname{diam}_{E}\left(J_{l, m}\right)\right)^{\alpha}=\sum_{l, m}\left(2^{\frac{1}{2}} \lambda^{k}\right)^{\alpha}=2^{\frac{\alpha}{2}} \lambda^{\alpha k} 2^{k}\left[\lambda^{-k}+1\right]<2^{\frac{\alpha+2}{2}}$.

Hence $\mathcal{H}_{E, \delta}^{\alpha}\left(A_{\alpha}\right)<2^{\frac{\alpha+2}{2}}$ for all $\delta>0$, so $\mathcal{H}_{E}^{\alpha}\left(A_{\alpha}\right) \leq 2^{\frac{\alpha+2}{2}}$.

The idea for proving $\mathcal{H}_{H}^{\alpha+1}\left(A_{\alpha}\right)>0$ is to apply Lemma 2.3 , i.e. to find a measure $\mu$ on $\left(\mathbb{H}, d_{H}\right)$ satisfying (i) and (ii).

Let $\mu=\mathcal{H}_{E}^{\alpha}$ be the $\alpha$-dimensional Hausdorff measure on $\mathbb{H} \equiv \mathbb{R}^{3}$ with respect to $d_{E}$. We must show that $\mu$ has the required properties: 
(i) We have $\mathcal{H}_{E}^{\alpha-1}\left(C_{\alpha-1}\right)>0$ (by Proposition 4.1 (ii)) and $\mathcal{H}_{E}^{1}(\{0\} \times$ $[0,1])=1$. Theorem $8.10(1)$ of $[\mathbf{M a}]$ gives

$$
\mu\left(A_{\alpha}\right)=\mathcal{H}_{E}^{(\alpha-1)+1}\left(C_{\alpha-1} \times\{0\} \times[0,1]\right)>0 .
$$

(ii) Let $p \in \mathbb{H}$ and $0<r<\frac{1}{4}$ such that $B_{H}(p, r) \cap(\mathbb{R} \times\{0\} \times$ $\mathbb{R}) \neq \emptyset$. Let $(x, 0, t),\left(x^{\prime}, 0, t^{\prime}\right) \in B_{H}(p, r) \cap(\mathbb{R} \times\{0\} \times \mathbb{R})$. Then $d_{H}\left((x, 0, t),\left(x^{\prime}, 0, t^{\prime}\right)\right) \leq 2 r$. In particular $\left|x^{\prime}-x\right| \leq 2 r$ and $\left|t^{\prime}-t\right| \leq 4 r^{2}$. Hence

$$
B_{H}(p, r) \cap A_{\alpha} \subseteq\left([x-2 r, x+2 r] \cap C_{\alpha-1}\right) \times\{0\} \times\left[t-4 r^{2}, t+4 r^{2}\right] .
$$

Let $k \in \mathbb{N}$ be minimal with $\lambda^{k} \leq 4 r$. Then $[x-2 r, x+2 r]$ intersects at most 3 intervals $I_{k, l}$, otherwise $[x-2 r, x+2 r]$ would have to contain some $I_{k-1, l}$. Thus $[x-2 r, x+2 r]$ intersects at most $3 \cdot 2^{k}$ intervals $I_{2 k, l}$. This gives

$$
\begin{aligned}
\mathcal{H}_{E}^{\alpha}(([x-2 r, x+2 r] & \left.\left.\cap C_{\alpha-1}\right) \times\{0\} \times\left[t-4 r^{2}, t+4 r^{2}\right]\right) \\
\leq & 3 \cdot 2^{k}\left(\left[8 r^{2} \lambda^{-2 k}\right]+1\right)\left(\operatorname{diam}_{E}\left(I_{k, l} \times\{0\} \times J\right)\right)^{\alpha},
\end{aligned}
$$

where $J$ is a subinterval of $\left[t-4 r^{2}, t+4 r^{2}\right]$ of diameter $\lambda^{2 k}$. Now

$$
\begin{aligned}
& 3 \cdot 2^{k}\left(\left[8 r^{2} \lambda^{-2 k}\right]+1\right)\left(\operatorname{diam}_{E}\left(I_{k, l} \times\{0\} \times J\right)\right)^{\alpha} \\
& \leq 9 \cdot 2^{k} 8 r^{2} \lambda^{-2 k}\left(2^{\frac{1}{2}} \lambda^{2 k}\right)^{\alpha}=9 \cdot 2^{k} 8 r^{2} \lambda^{-2 k} 2^{\frac{\alpha}{2}}\left(\lambda^{2 k}\right)^{\alpha-1} \lambda^{2 k} \\
& \quad=K_{1} 2^{k} 8 r^{2}\left(\lambda^{k}\right)^{\alpha-1}\left(\lambda^{k}\right)^{\alpha-1}=K_{2} 8 r^{2}\left(\lambda^{k}\right)^{\alpha-1} \leq K_{3} r^{\alpha+1}
\end{aligned}
$$

with $K_{1}, K_{2}, K_{3}>0$ not depending on $r$. We have used $1 \leq 2 \cdot 8 r^{2} \lambda^{-2 k}$, $\lambda^{\alpha-1}=\frac{1}{2}$ and $\lambda^{k} \leq 4 r$. Summing up we get

$$
\mu\left(B_{H}(p, r) \cap A_{\alpha}\right) \leq c_{\mu} r^{\alpha+1}
$$

with a positive constant $c_{\mu}$ not depending on $r$.

3. Case $\boldsymbol{\alpha}=\mathbf{2}$. In this case, the argument is based on Pansu's isoperimetric inequality (see $[\mathbf{P a 2}]$ ), which says that if $\Omega \subseteq \mathbb{H}$ is a bounded domain with piecewise $C^{2}$ smooth boundary $\partial \Omega$, then

$$
\mathcal{H}_{H}^{3}(\partial \Omega) \geq c\left(\mathcal{L}^{3}(\Omega)\right)^{\frac{3}{4}}
$$

with some absolute constant $c>0$. Now, setting $A_{2}:=\partial B_{E}(0,1)$ and using (4.1), we obtain $\mathcal{H}_{H}^{3}\left(A_{2}\right)>0$ as required. 
4. Case $2<\boldsymbol{\alpha}<\mathbf{3}$. The set $A_{\alpha}$ in this case is a Cantor-set of spheres:

$$
A_{\alpha}:=\bigcup_{r \in C_{\alpha-2}} S_{H}(0, r),
$$

where $S_{H}(0, r)=\left\{p \in \mathbb{H} \mid\|p\|_{H}=r\right\}$ is the sphere of radius $r$ centered at 0 with respect to $d_{H}$ and $C_{\alpha-2}$ is the Cantor-set of Hausdorff dimension $\alpha-2$. The estimate $\mathcal{H}_{E}^{\alpha}\left(A_{\alpha}\right)<\infty$ follows from the product type structure of $A_{\alpha} . A_{\alpha}$ is not directly a product set, but it is the image of a product-type set under a Lipchitz mapping. The proof is then similar to the proof in Case 2. The details can be found in [Ri]. The set $A_{\alpha}$ appeared already in [Ba1] in connection with quasiconformal mappings of the Heisenberg group. Using Pansu's isoperimetric inequality, it was shown (see Lemma 5.2 and the subsequent remark) that $\mathcal{H}_{H, \infty}^{\alpha+1}\left(A_{\alpha}\right)>0$.

5. Case $\boldsymbol{\alpha}=$ 3. Simply take $A_{3}:=B_{H}(0,1)$. Then $0<\mathcal{H}_{E}^{3}\left(A_{3}\right)<\infty$ and $0<\mathcal{H}_{H}^{4}\left(A_{3}\right)<\infty$.

\subsection{Sharpness of $\mathcal{H}_{E}^{\min \left\{\alpha, 1+\frac{\alpha}{2}\right\}} \ll \mathcal{H}_{H}^{\alpha}$.}

Let us remember that in order to show the sharpness of $\mathcal{H}_{E}^{\min \left\{\alpha, 1+\frac{\alpha}{2}\right\}} \ll$ $\mathcal{H}_{H}^{\alpha}$, we have to construct sets which are in some sense tangent to the horizontal distribution. For small dimensions $\alpha$ this is fairly easy: we just consider subsets of horizontal curves. For higher dimensions things become more complicated and we have to use some recent results obtained in $[\mathbf{B a 2}]$ about the so-called characteristic sets.

1. Case $0<\boldsymbol{\alpha} \leq \mathbf{1}$. The choice of the set $A_{\alpha}$ in the case $0<\alpha \leq 1$ is based on the fact that the restriction of $d_{H}$ to horizontal curves is bi-Lipschitz equivalent to $d_{E}$. Observe that the $O_{x}$ axis is a horizontal curve. In fact, the restrictions of $d_{H}$ and $d_{E}$ to $O_{x}$ coincide. Hence, if we let $A_{\alpha}:=\left\{(x, 0,0) \mid x \in C_{\alpha}\right\}$ when $0<\alpha<1$ and $A_{1}:=\{(x, 0,0) \mid$ $x \in[0,1]\}$, then $0<\mathcal{H}_{E}^{\alpha}\left(A_{\alpha}\right)=\mathcal{H}_{H}^{\alpha}\left(A_{\alpha}\right)<\infty$ by Propositions 2.2 (iv) and 4.1 (ii).

The construction of the sets $A_{\alpha}$, respectively $A_{\alpha, \delta}$, in the remaining cases is more involved. We start with a definition:

Definition 4.1. Let $S \subseteq \mathbb{R}^{3}$ be a $C^{1}$ smooth, regular surface. A point $p \in$ $S$ is a characteristic point for $S$ if $\mathrm{T}_{p} S=\mathrm{H}_{p} \mathbb{H}$, that is if the tangent space of $S$ and the horizontal plane coincide at $p$. The characteristic set $C(S)$ of $S$ is the set of characteristic points for $S$. 
Remark 4.1. Assume that the surface $S$ is given as the graph of a $C^{1}$ function $f: U \rightarrow \mathbb{R}$ over a domain $U \subseteq \mathbb{R}^{2}$, i.e. $S=\{(x, y, f(x, y))$ $(x, y) \in U\}$. Then $p=(x, y, t) \in S$ is a characteristic point if and only if

$$
\frac{\partial f}{\partial x}(x, y)=2 y \quad \text { and } \quad \frac{\partial f}{\partial y}(x, y)=-2 x .
$$

In what follows we consider characteristic sets $C(S)$ on such graphs. We collect the results from $[\mathbf{B a 2}]$ needed in the sequel in the following theorem:

Theorem 4.2. Given $1<\alpha<2$, there exists a compact subset $Q_{\alpha}$ of $Q:=\left[-\frac{1}{2}, \frac{1}{2}\right] \times\left[-\frac{1}{2}, \frac{1}{2}\right]$ and a $C^{1,1}$ smooth map $f: Q \rightarrow \mathbb{R}$, such that:

(i) $0<\mathcal{H}_{E}^{\alpha}\left(Q_{\alpha}\right)<\infty$.

(ii) $\left\{(x, y, f(x, y)) \mid(x, y) \in Q_{\alpha}\right\} \subseteq C(S)$, where $S=\{(x, y, f(x, y)) \mid$ $(x, y) \in Q\}$.

(iii) For any $s \geq 0$, there is a constant $c=c(s)$ such that $\mathcal{H}_{H}^{s}(A) \leq$ $c \mathcal{H}_{E}^{s}(A)$ whenever $A \subseteq C(S)$.

Remark 4.2. (i) and (ii) are shown in the proof of Theorem 1.4 in [Ba2]. (iii) is essentially Case 2 of Theorem 1.1 in [Ba2] where $\mathcal{H}_{H, \infty}^{t}$ (respectively $\mathcal{H}_{E, \infty}^{t}$ ) has been replaced by $\mathcal{H}_{H}^{t}$ (respectively $\mathcal{H}_{E}^{t}$ ). The proof given there only needs very minor changes in our case. The computations can be found in $[\mathbf{R i}]$.

2. Case $\mathbf{1}<\boldsymbol{\alpha}<\mathbf{2}$. Our set $A_{\alpha}$ is obtained from the characteristic set of a surface in the following way: Let $Q_{\alpha}$ and $f: Q \rightarrow \mathbb{R}$ be as in Theorem 4.2. Consider the mapping $F: Q \rightarrow \mathbb{R}^{3}$ given by $F(x, y)=$ $(x, y, f(x, y))$ and set $A_{\alpha}:=F\left(Q_{\alpha}\right)$. Observe that $F:\left(Q_{\alpha}, d_{E}\right) \rightarrow$ $\left(A_{\alpha}, d_{E}\right)$ is a bi-Lipschitz mapping and therefore

$$
\frac{1}{k} \mathcal{H}_{E}^{\alpha}\left(Q_{\alpha}\right) \leq \mathcal{H}_{E}^{\alpha}\left(A_{\alpha}\right) \leq k \mathcal{H}_{E}^{\alpha}\left(Q_{\alpha}\right)
$$

for some $k>0$. This shows in particular that $\mathcal{H}_{E}^{\alpha}\left(A_{\alpha}\right)>0$. We apply Theorem 4.2 to estimate $\mathcal{H}_{H}^{\alpha}\left(A_{\alpha}\right)$. Since $A_{\alpha} \subseteq C(S)$, it follows that

$$
\mathcal{H}_{H}^{\alpha}\left(A_{\alpha}\right) \leq c \mathcal{H}_{E}^{\alpha}\left(A_{\alpha}\right) \leq c k \mathcal{H}_{E}^{\alpha}\left(Q_{\alpha}\right)
$$

3. Case $\boldsymbol{\alpha}=2$. Given $0<\delta<2$ arbitrary, consider the set $Q_{2-\delta}$ and the map $F$ from the Case 2 above. Then for $A_{\alpha, \delta}:=F\left(Q_{2-\delta}\right)$, we have $\mathcal{H}_{E}^{2-\delta}\left(A_{\alpha, \delta}\right)>0$ and $\mathcal{H}_{H}^{2}\left(A_{\alpha, \delta}\right)=0$ by Theorem 4.2 . 
4. Case $2<\boldsymbol{\alpha}<\mathbf{4}$. Given $0<\delta<1$ arbitrary, consider the map $F$ and the set $F\left(Q_{2-\delta}\right)$ from 2. above. Our set $A_{\alpha, \delta}$ will be a product of $F\left(Q_{2-\delta}\right)$ with the Cantor set $C_{\frac{\alpha-2}{2}}$ lying in the vertical axis:

$$
A_{\alpha, \delta}:=\left\{(x, y, f(x, y)+t) \mid(x, y) \in Q_{2-\delta}, t \in C_{\frac{\alpha-2}{2}}\right\} .
$$

The estimate $\mathcal{H}_{E}^{1+\frac{\alpha}{2}-\delta}\left(A_{\alpha, \delta}\right)>0$ is again a consequence of the Euclidean product structure of $A_{\alpha, \delta}$. To see this, consider the map

$$
g: Q_{2-\delta} \times C_{\frac{\alpha-2}{2}} \rightarrow A_{\alpha, \delta} ; \quad g(x, y, t):=F(x, y)+(0,0, t) .
$$

$g$ is a bijection, and the inverse is

$$
g^{-1}: A_{\alpha, \delta} \rightarrow Q_{2-\delta} \times C_{\frac{\alpha-2}{2}} ; \quad g^{-1}(x, y, t):=(x, y, t)-(0,0, f(x, y)) .
$$

It is easy to see that $g:\left(Q_{2-\delta} \times C_{\frac{\alpha-2}{2}}, d_{E}\right) \rightarrow\left(A_{\alpha, \delta}, d_{E}\right)$ is bi-Lipschitz. Hence, it is enough to show that

$$
\mathcal{H}_{E}^{1+\frac{\alpha}{2}-\delta}\left(Q_{2-\delta} \times C_{\frac{\alpha-2}{2}}\right)>0 .
$$

But $\mathcal{H}_{E}^{2-\delta}\left(Q_{2-\delta}\right)>0$ and $\mathcal{H}_{E}^{\frac{\alpha-2}{2}}\left(C_{\frac{\alpha-2}{2}}\right)>0$, by Proposition 4.1 (ii) and Theorem 4.2, so

$$
\mathcal{H}_{E}^{1+\frac{\alpha}{2}-\delta}\left(Q_{2-\delta} \times C_{\frac{\alpha-2}{2}}\right)=\mathcal{H}_{E}^{(2-\delta)+\left(\frac{\alpha-2}{2}\right)}\left(Q_{2-\delta} \times C_{\frac{\alpha-2}{2}}\right)>0
$$

by [Ma, Theorem 8.10 (1)].

Next, we prove that $\mathcal{H}_{H, \infty}^{\alpha}\left(A_{\alpha, \delta}\right)=0$. This is a more difficult task since we have to consider coverings with Heisenberg balls. In our argument it is crucial that the shape of Heisenberg balls remains unchanged under vertical translations. Now remember that $0<\lambda<1$ is related to $C_{\frac{\alpha-2}{2}}$ by the equation $\lambda^{\frac{\alpha-2}{2}}=\frac{1}{2}$ and let $c:=\left(1+\lambda^{-\frac{1}{2}}\right)^{2} 2^{\alpha-2}$. Given $0<\epsilon<c \lambda^{2}$, using the compactness of $F\left(Q_{2-\delta}\right)$ and the fact that $\mathcal{H}_{H}^{2}\left(F\left(Q_{2-\delta}\right)\right)=0$, we can cover $F\left(Q_{2-\delta}\right)$ with Heisenberg balls $\left(B_{H}\left(p_{n}, r_{n}\right)\right)_{n \in\{1, \ldots, N\}}$ in such a way that $\sum_{n=1}^{N}\left(2 r_{n}\right)^{2}<\epsilon / c$.

For each $n \in\{1, \ldots, N\}$, let $k_{n} \in \mathbb{N}$ such that $\lambda^{\frac{k_{n}+1}{2}} \leq 2 r_{n}<\lambda^{\frac{k_{n}}{2}}$ $\left(2 r_{n}<(\epsilon / c)^{\frac{1}{2}}<\lambda\right.$ for $n=1, \ldots, N$ by construction). For $n=1, \ldots, N$ and $l_{n}=1, \ldots, 2^{k_{n}}$, let

$$
B_{n, l_{n}}:=\left\{\left(x, y, t+t^{\prime}\right) \mid(x, y, t) \in B_{H}\left(p_{n}, r_{n}\right), t^{\prime} \in I_{k_{n}, l_{n}}\right\},
$$

where $I_{k_{n}, l_{n}}$ are the intervals appearing in the construction of the Cantor set $C_{\frac{\alpha-2}{2}}$. Notice that $A_{\alpha, \delta} \subseteq \bigcup_{n, l_{n}} B_{n, l_{n}}$. In what follows we estimate 
the Heisenberg diameter of $B_{n, l_{n}}$ : Let $(x, y, t),\left(x^{\prime}, y^{\prime}, t^{\prime}\right) \in B_{H}\left(p_{n}, r_{n}\right)$ and let $t_{1}, t_{2} \in I_{k_{n}, l_{n}}$. Then

$$
\begin{aligned}
d_{H} & \left(\left(x, y, t+t_{1}\right),\left(x^{\prime}, y^{\prime}, t^{\prime}+t_{2}\right)\right) \\
& =\left\|\left(x^{\prime}-x, y^{\prime}-y,\left(t^{\prime}-t\right)+\left(t_{2}-t_{1}\right)-2 x^{\prime} y+2 x y^{\prime}\right)\right\|_{H} \\
& =\left\|\left(x^{\prime}-x, y^{\prime}-y,\left(t^{\prime}-t\right)-2 x^{\prime} y+2 x y^{\prime}\right) *\left(0,0, t_{2}-t_{1}\right)\right\|_{H} \\
& \leq d_{H}\left((x, y, t),\left(x^{\prime}, y^{\prime}, t^{\prime}\right)\right)+\left|t_{2}-t_{1}\right|^{\frac{1}{2}} \\
& \leq 2 r_{n}+\lambda^{\frac{k_{n}}{2}} .
\end{aligned}
$$

This computation gives $\operatorname{diam}_{H}\left(B_{n, l_{n}}\right) \leq 2 r_{n}+\lambda^{\frac{k_{n}}{2}}$. Now:

$$
\begin{aligned}
& \sum_{n, l_{n}}\left(\operatorname{diam}_{H}\left(B_{n, l_{n}}\right)\right)^{\alpha} \\
& \quad=\sum_{n, l_{n}}\left(\operatorname{diam}_{H}\left(B_{n, l_{n}}\right)\right)^{2}\left(\operatorname{diam}_{H}\left(B_{n, l_{n}}\right)\right)^{\alpha-2} \\
& \quad \leq \sum_{n, l_{n}}\left(2 r_{n}+\lambda^{\frac{k_{n}}{2}}\right)^{2}\left(2 r_{n}+\lambda^{\frac{k_{n}}{2}}\right)^{\alpha-2} \leq \sum_{n, l_{n}}\left(\left(1+\lambda^{-\frac{1}{2}}\right) 2 r_{n}\right)^{2}\left(2 \lambda^{\frac{k_{n}}{2}}\right)^{\alpha-2} \\
& \quad=\sum_{n, l_{n}}\left(1+\lambda^{-\frac{1}{2}}\right)^{2}\left(2 r_{n}\right)^{2} 2^{\alpha-2}\left(\frac{1}{2}\right)^{k_{n}}=\sum_{n}\left(1+\lambda^{-\frac{1}{2}}\right)^{2}\left(2 r_{n}\right)^{2} 2^{\alpha-2} \\
& \quad=\left(1+\lambda^{-\frac{1}{2}}\right)^{2} 2^{\alpha-2} \sum_{n}\left(2 r_{n}\right)^{2}=c \sum_{n}\left(2 r_{n}\right)^{2}<c \frac{\epsilon}{c}=\epsilon .
\end{aligned}
$$

Hence, for an arbitrary $0<\epsilon<c \lambda^{2}$, there exists a covering $\left(B_{n, l_{n}}\right)_{n, l_{n}}$ $\left(n=1, \ldots, N, l_{n}=1, \ldots, 2^{k_{n}}\right)$ of $A_{\alpha, \delta}$ such that $\sum_{n, l_{n}}\left(\operatorname{diam}_{H}\left(B_{n, l_{n}}\right)\right)^{\alpha}<$ $\epsilon$.

5. Case $\boldsymbol{\alpha}=4$. This, again, is trivial. Take $A_{4}:=B_{E}(0,1)$. Then $0<\mathcal{H}_{E}^{3}\left(A_{4}\right)<\infty$ and $0<\mathcal{H}_{H}^{4}\left(A_{4}\right)<\infty$.

\section{An application to $\mathbb{H}$-rectifiability}

In this section we will compare the classical notion of 2-Euclidean rectifiability and the notion of $\mathbb{H}$-rectifiability introduced in [FSSC1].

Let us recall the classical notion of $m$-rectifiability in a general metric space due to Federer $([\mathbf{F e}, 3.2 .14])$ : Given a metric space $(X, d)$ and a 
positive integer $m$, we say that a Borel set $E \subseteq X$ is $m$-rectifiable if there exists a countable collection of Lipschitz maps $f_{i}: A_{i} \subseteq\left(\mathbb{R}^{m}, d_{E}\right) \rightarrow$ $(X, d)$ such that

$$
\mathcal{H}_{d}^{m}\left(E \backslash \bigcup_{i=1}^{\infty} f_{i}\left(A_{i}\right)\right)=0 .
$$

An $m$-dimensional Euclidean rectifiable set is an $m$-rectifiable subset of $\left(\mathbb{R}^{n}, d_{E}\right)$.

Remark 5.1. It is well known that $m$-dimensional Euclidean rectifiability is equivalent to the requirement that $\mathcal{H}_{d_{E}}^{m}$-almost all of the set can be covered by a sequence of $C^{1} m$-graphs of $\mathbb{R}^{n}$ (see [AFP, Chapter 2, Section 2.9]).

It turned out however that this notion of rectifiability is not appropriate in the setting of the Heisenberg group endowed with the Heisenberg metric. Indeed, Ambrosio and Kirchheim (see [AK, Theorem 7.2]) proved that the Heisenberg group $\left(\mathbb{H}, d_{H}\right)$ is purely $m$-unrectifiable for $m=2,3,4$, i.e. that for any Lipschitz map

$$
f: A \subseteq\left(\mathbb{R}^{m}, d_{E}\right) \rightarrow\left(\mathbb{H}, d_{H}\right)
$$

one has $\mathcal{H}_{H}^{m}(f(A))=0$. This lack of rectifiable sets in the classical sense suggests that more intrinsic definitions of rectifiability could be useful instead. To this aim, in [FSSC1], an intrinsic definition of rectifiability in the Heisenberg group was introduced in the codimension one case. This was successfully used to study the structure of the sets of intrinsic finite perimeter (see [FSSC1], [FSSC2], [FSSC3]).

The idea in [FSSC1] was to replace the images of Lipschitz mappings in Federer's definition by surfaces given as level sets of $\mathbb{H}$-differentiable functions. This led to the following definition:

Definition 5.1. We shall say that $S \subseteq \mathbb{H}$ is 3-dimensional $\mathbb{H}$-rectifiable if there exists a sequence of $\mathbb{H}$-regular hypersurfaces $\left(S_{i}\right)_{i \in \mathbb{N}}$ such that

$$
\mathcal{H}_{H}^{3}\left(S \backslash \bigcup_{i \in \mathbb{N}} S_{i}\right)=0 .
$$

In the above definition the notion of $\mathbb{H}$-regular hypersurface appears. Here $S \subseteq \mathbb{R}^{3} \equiv \mathbb{H}$ is called an $\mathbb{H}$-regular hypersurface if it is locally the level set of an $\mathbb{H}$-regular function $f: \mathbb{H} \rightarrow \mathbb{R}$. $\mathbb{H}$-regularity of $f$ means that the horizontal gradient

$$
\nabla_{\mathbb{H}} f:=(X f, Y f): \mathbb{H} \rightarrow \mathbb{R}^{2}
$$


of $f$ exists, is continuous and non-vanishing on $S$. In particular, if $S$ is a Euclidean $C^{1}$ regular surface without characteristic points, then $S$ is $\mathbb{H}$-regular.

It was proved in $[\mathbf{F S S C 1}]$ that the essential boundary of a set $E$ of locally finite $\mathbb{H}$-perimeter in $\mathbb{H}$ is 3 -dimensional $\mathbb{H}$-rectifiable.

The following comparison result between 2-dimensional Euclidean rectifiability and 3-dimensional $\mathbb{H}-$-rectifiability is in order:

\section{Theorem 5.1.}

(i) Each 2-dimensional Euclidean rectifiable set $S \subseteq \mathbb{R}^{3} \equiv \mathbb{H}$ is 3-dimensional $\mathbb{H}$-rectifiable.

(ii) There are 3 -dimensional $\mathbb{H}$-rectifiable sets $S \subseteq \mathbb{R}^{3} \equiv \mathbb{H}$ that are not 2-dimensional Euclidean rectifiable.

Proof: (i) By Remark 5.1, given a 2-dimensional Euclidean rectifiable subset $S \subseteq \mathbb{R}^{3}$, we can assume without loss of generality that

$$
S=N \cup \bigcup_{i \in \mathbb{N}} S_{i}
$$

where $\mathcal{H}_{E}^{2}(N)=0$ and $S_{i} \subseteq \mathbb{R}^{3}$ is a Euclidean $C^{1}$ regular hypersurface. Let $\tilde{S}_{i}:=S_{i} \backslash C\left(S_{i}\right)$, where $C\left(S_{i}\right)$ denotes the set of characteristic points of $S_{i}$. Then $\tilde{S}_{i}$ is an $\mathbb{H}$-regular hypersurface, and

$$
S=\tilde{N} \cup \bigcup_{i \in \mathbb{N}} \tilde{S}_{i}
$$

with

$$
\tilde{N}=N \cup \bigcup_{i \in \mathbb{N}} C\left(S_{i}\right) .
$$

By Theorem 1.1 with $\alpha=2$ we have $\mathcal{H}_{H}^{3}(N)=0$. According to a result from [Ba2], if $S$ is a Euclidean $C^{1}$ regular hypersurface, then $\mathcal{H}_{H}^{3}(C(S))=0$. This gives $\mathcal{H}_{H}^{3}\left(C\left(S_{i}\right)\right)=0$ for all $i \in \mathbb{N}$. Hence $\mathcal{H}_{H}^{3}(\tilde{N})=0$ and consequently $S$ is 3 -dimensional $\mathbb{H}$-rectifiable.

(ii) Given $0<\delta<0.5$, we know by Theorem 1.2 (ii) that there is a compact set $N=A_{3, \delta} \subseteq \mathbb{H}$ such that

$$
\mathcal{H}_{H}^{3}(N)=0 \text { but } \mathcal{H}_{E}^{2.5-\delta}(N)>0 .
$$

Therefore $N$ is 3-dimensional $\mathbb{H}$-rectifiable by definition but not 2-dimensional Euclidean rectifiable since its Hausdorff dimension in $\left(\mathbb{R}^{3}, d_{E}\right)$ is strictly bigger than 2 . 


\section{References}

[AFP] L. Ambrosio, N. Fusco and D. Pallara, "Functions of bounded variation and free discontinuity problems", Oxford Mathematical Monographs, The Clarendon Press, Oxford University Press, New York, 2000.

[AK] L. Ambrosio And B. Kirchineim, Rectifiable sets in metric and Banach spaces, Math. Ann. 318(3) (2000), 527-555.

[Ba1] Z. M. BALOGH, Hausdorff dimension distribution of quasiconformal mappings on the Heisenberg group, J. Anal. Math. $\mathbf{8 3}$ (2001), 289-312.

[Ba2] Z. M. BALOGH, Size of characteristic sets and functions with prescribed gradient, Preprint.

[Be] A. Bellaïche, The tangent space in sub-Riemannian geometry, in: "Sub-Riemannian geometry", Progr. Math. 144, Birkhäuser, Basel, 1996, pp. 1-78.

[Cy] J. Cygan, Subadditivity of homogeneous norms on certain nilpotent Lie groups, Proc. Amer. Math. Soc. 83(1) (1981), 69-70.

[DS] G. David And S. Semmes, "Fractured fractals and broken dreams. Self-similar geometry through metric and measure", Oxford Lecture Series in Mathematics and its Applications 7, The Clarendon Press, Oxford University Press, New York, 1997.

[Fa] K. J. FALCONER, "The geometry of fractal sets", Cambridge Tracts in Mathematics 85, Cambridge University Press, Cambridge, 1986.

[Fe] H. FeDERER, "Geometric measure theory", Die Grundlehren der mathematischen Wissenschaften 153, Springer-Verlag New York Inc., New York, 1969.

[FoSt] G. B. Folland And E. M. Stein, "Hardy spaces on homogeneous groups", Mathematical Notes 28, Princeton University Press, Princeton, N.J.; University of Tokyo Press, Tokyo, 1982.

[FSSC1] B. Franchi, R. Serapioni and F. Serra Cassano, Rectifiability and perimeter in the Heisenberg group, Math. Ann. 321(3) (2001), 479-531.

[FSSC2] B. Franchi, R. Serapioni and F. Serra Cassano, Regular hypersurfaces, intrinsic perimeter and implicit function theorem in Carnot groups, Comm. Anal. Geom. (to appear). 
[FSSC3] B. Franchi, R. Serapioni and F. Serra Cassano, Rectifiability and perimeter in step 2 groups, Math. Bohem. (to appear).

[Fre] D. H. Fremlin, Spaces of finite length, Proc. London Math. Soc. (3) 64(3) (1992), 449-486.

[Gro] M. Gromov, Carnot-Carathéodory spaces seen from within, in: "Sub-Riemannian geometry", Progr. Math. 144, Birkhäuser, Basel, 1996, pp. 79-323.

[HK] P. Hajlasz and P. Koskela, Sobolev met Poincaré, Mem. Amer. Math. Soc. 145(688) (2000), 101 pp.

[How] J. D. Howroyd, On dimension and on the existence of sets of finite positive Hausdorff measure, Proc. London Math. Soc. (3) 70(3) (1995), 581-604.

[K] B. KirchHeIm, Rectifiable metric spaces: local structure and regularity of the Hausdorff measure, Proc. Amer. Math. Soc. 121(1) (1994), 113-123.

[Ma] P. Mattila, "Geometry of sets and measures in Euclidean spaces. Fractals and rectifiability", Cambridge Studies in Advanced Mathematics 44, Cambridge University Press, Cambridge, 1995 .

[Mitc] J. Mitchell, On Carnot-Carathéodory metrics, J. Differential Geom. 21(1) (1985), 35-45.

[Pa1] P. Pansu, Géometrie du groupe d'Heisenberg, Thèse pour le titre de Docteur troisième cycle, Université Paris VII (1982).

[Pa2] P. Pansu, Une inégalité isopérimétrique sur le groupe de Heisenberg, C. R. Acad. Sci. Paris Sér. I Math. 295(2) (1982), 127-130.

[Pau] S. D. PAuls, A notion of rectifiability modelled on Carnot groups, Preliminary announcement (2000).

[PT] D. Preiss And J. TišER, On Besicovitch's $\frac{1}{2}$-problem, J. London Math. Soc. (2) 45(2) (1992), 279-287.

[Ri] M. RICKLY, Invariant metrics and Hausdorff measures on the Heisenberg group, Thesis for the master degree, University of Bern (2002).

[Se] S. Semmes, On the nonexistence of bi-Lipschitz parameterizations and geometric problems about $A_{\infty}$-weights, Rev. Mat. Iberoamericana 12(2) (1996), 337-410.

[Str] R. S. StrichartZ, Self-similarity on nilpotent Lie groups, in: "Geometric analysis" (Philadelphia, PA, 1991), Contemp. Math. 140, Amer. Math. Soc., Providence, RI, 1992, pp. $123-157$. 
Zoltán M. Balogh:

Institute of Mathematics

University of Bern

Sidlerstrasse 5

3012 Bern

Switzerland

E-mail address: zoltan.balogh@math-stat.unibe.ch

Matthieu Rickly:

Institute of Mathematics

University of Bern

Sidlerstrasse 5

3012 Bern

Switzerland

E-mail address: matthieu.rickly@math-stat.unibe.ch

Francesco Serra Cassano:

Dipartimento di Matematica

Università di Trento

Via Sommarive 14

38050 Povo (Trento)

Italy

E-mail address: cassano@science.unitn.it

Rebut el 4 de juny de 2002. 